\title{
Thermal quenching at the microscopic level in multi-phase thiosilicate phosphors
}

\author{
Philippe F. Smet, Jonas Botterman, Anthony B. Parmentier, Dirk Poelman
}

this paper was published in Optical Materials 35 (2013) 1970-1975

DOI: 10.1016/j.optmat.2012.10.051

the version below is the final submitted version, before copy-editing

Contact details:

dr. Philippe Smet, LumiLab,

Department of Solid State Sciences, Ghent University, Gent, Belgium.

T: +32 92644353

philippe.smet@ugent.be http://lumilab.ugent.be 


\title{
Thermal quenching at the microscopic level in
}

\section{multi-phase thiosilicate phosphors}

\author{
Philippe F. Smet ${ }^{1}$, Jonas Botterman, Anthony B. Parmentier, Dirk Poelman \\ LumiLab, Department of Solid State Sciences, Ghent University, Ghent, Belgium. \\ Center for Nano- and Biophotonics (NB Photonics), Ghent University, Ghent, Belgium. \\ 1philippe.smet@ugent.be
}

\begin{abstract}
.
Europium $\left(\mathrm{Eu}^{2+}\right)$ doped calcium thiosilicate $\left(\mathrm{Ca}_{2} \mathrm{SiS}_{4}\right)$ is interesting as conversion phosphor for light-emitting diodes (LEDs), because of its yellow-to-red emission, depending on the europium concentration. However, for elevated europium concentrations two phases appear which is reflected in the temperature dependency of the emission spectrum. In this work we study the emission spectrum and thermal quenching behaviour at the microscopic level, by performing temperature dependent cathodoluminescence spectroscopy in a scanning electron microscope. A clear relation between the local europium concentration and the thermal quenching is obtained. This proves useful to explain the luminescence properties of the bulk phosphor.
\end{abstract}

Keywords: cathodoluminescence, thiosilicate, dopant distribution, europium, thermal quenching. 


\section{Introduction.}

White light-emitting diodes (LEDs) are on the verge of replacing incandescent and fluorescent lamps due to their small footprint, high efficiency and long lifetime $[1,2]$. The replacement occurs in the field of general lighting, as well as for display back lighting. In general, white LEDs are comprised of a blue emitting LED chip and one or more phosphor materials which convert part of the blue light to longer wavelengths. Current research efforts are focussed on more lumen efficient devices and improved colour rendering. In that respect, special attention is paid to the development of efficient red emitting phosphor materials, with relatively narrow emission bands to improve both colour saturation and lumen output. Phosphors doped with divalent europium are a prime choice, if hosts with a sufficiently high red-shift - being the combination of the nephelauxetic effect, the crystal field splitting and the Stokes shift - can be obtained [3]. After the initial use of alkaline earth sulfide phosphors, most orange-to-red LED phosphors are currently based on chemically stable nitrides, such as $\mathrm{Sr}_{2} \mathrm{Si}_{5} \mathrm{~N}_{8}$ :Eu or $\mathrm{CaAlSiN}_{3}$ :Eu $[1,2,4-7]$.

The alkaline earth thiosilicate phosphors, with general formula $\mathrm{M}_{1-x} \mathrm{RE}_{x} \mathrm{Si}_{\mathrm{y}} \mathrm{S}_{2 \mathrm{y}+1}\left(\mathrm{M}=\mathrm{Ca}^{2+}, \mathrm{Sr}^{2+}\right.$, $\mathrm{Ba}^{2+}, \mathrm{RE}=$ rare earth element, e.g. $\mathrm{Eu}^{2+}$ or $\left.\mathrm{Ce}^{3+}\right)$, are nevertheless an interesting class of sulfide phosphor materials [8-10]. The emission ranges from bluish-green $\left(\mathrm{Ba}_{2} \mathrm{SiS}_{4}: \mathrm{Eu}[9,11]\right)$, green $\left(\mathrm{Sr}_{2} \mathrm{SiS}_{4}: \mathrm{Eu}\right.$ [12]) to red $\left(\mathrm{Ca}_{2} \mathrm{SiS}_{4}: \mathrm{Eu}\right.$ [13]) upon doping with divalent europium [14]. In general, the concentration quenching in the case of $\mathrm{Eu}^{2+}$-doped thiosilicates is relatively limited, and high dopant concentrations can be introduced into the lattice, which is beneficial to obtain strong absorption of the excitation light. Note that also the stoichiometric phosphor $\mathrm{Eu}_{2} \mathrm{SiS}_{4}$ is still luminescent at room temperature, although its thermal quenching is rather severe $[13,15,16]$. While the similarity in ionic radii for $\mathrm{Eu}^{2+}$ and 
$\mathrm{Sr}^{2+}$ leads to full miscibility in the $\mathrm{Sr}_{2-x} \mathrm{Eu}_{x} \mathrm{SiS}_{4}$ system, $\mathrm{Ca}_{2} \mathrm{SiS}_{4}$ and $\mathrm{Eu}_{2} \mathrm{SiS}_{4}$ have a different crystallographic structure (orthorhombic and monoclinic, respectively) [13]. In earlier work, it was noticed that a substitution of $\mathrm{Ca}$ by Eu in the range of 15 to $40 \%$ does not lead to a single phase material, but to a mixture of $\mathrm{Ca}_{2} \mathrm{SiS}_{4}$ - and $\mathrm{Eu}_{2} \mathrm{SiS}_{4}$-like phosphors [13].

One of the key requirements for conversion phosphors in light-emitting diodes (LEDs) is the temperature dependency of the luminescence [1]. In most LED designs, the conversion phosphor is situated close to the pumping LED. As a consequence, the phosphor is operated considerably above ambient temperature due to the transfer of heat generated in the LED chip, non-radiative transitions and Stokes losses (or the quantum deficit) in the phosphor. Therefore, it is important to know how temperature affects the luminescence properties of the phosphor, both in terms of efficiency (i.e. the thermal quenching) and colour changes (i.e. due to shifts in the position and shape of the emission spectrum). From an application point of view, it is sufficient to measure and evaluate this behaviour on a macroscopic level, as in a device the light emitted from the individual phosphor particles is blended anyway. However, from a scientific point of view, the underlying distribution of emission properties at the single particle level is important. In this way, non-uniform doping or impurity phases can be identified. This can then be used to improve synthesis conditions and possibly also the performance of the phosphor as a whole.

An interesting technique to study the luminescence at a microscopic level is SEM-(EDX-)CL. In this method, the imaging capabilities of a scanning electron microscope (SEM) are combined with cathodoluminescence $(C L)$ spectroscopy. Energy-dispersive $x$-ray analysis (EDX) allows adding information on the chemical composition. SEM-(EDX)-CL is applied in a wide range of scientific fields, from defect characterization in semiconductors [17], over 
geological studies on meteorite impact deformations in quartz [18], to high resolution biological imaging [19]. The technique was recently used to study the miscibility of $(\mathrm{Ca}, \mathrm{Sr})_{2} \mathrm{SiS}_{4}: \mathrm{Eu}$ phosphors [12]. In the present work, a temperature stage $\left(-25^{\circ} \mathrm{C}\right.$ to $\left.150^{\circ} \mathrm{C}\right)$ was used to probe the thermal quenching behaviour at the microscopic level, both in terms of emission spectrum and intensity. This is in contrast to common measurements of the thermal quenching, which measure bulk phosphors and lack spatial resolution. We focused on a phosphor with high europium concentration, namely $\mathrm{Ca}_{2} \mathrm{SiS}_{4}$ with $25 \%$ of the $\mathrm{Ca}^{2+}$ ions replaced by $\mathrm{Eu}^{2+}$. Although the intended composition is $\mathrm{Ca}_{1.5} \mathrm{Eu}_{0.5} \mathrm{SiS}_{4}$, it will be shown that a mixture of two luminescent phases is obtained.

\section{Experimental.}

The phosphor with intended composition $\mathrm{Ca}_{1.5} \mathrm{Eu}_{0.5} \mathrm{SiS}_{4}$ was synthesized from a mixture of CaS, EuS and Si (in slight excess) under a flow of $\mathrm{H}_{2} \mathrm{~S}$ at $900^{\circ} \mathrm{C}$. It shows an orange body colour due to the strong absorption for wavelengths up to $550 \mathrm{~nm}$. More details on the synthesis method can be found elsewhere [13]. After grinding, the crystallographic structure of the phosphor was evaluated using x-ray diffraction (XRD) with a Bruker D5000 diffractometer, operated in $\theta-2 \theta$ mode and by means of $\mathrm{Cu}-\mathrm{K} \alpha$ radiation $(\lambda=0.154 \mathrm{~nm}, 40 \mathrm{kV}$, $40 \mathrm{~mA})$.

SEM-EDX-CL measurements were performed in a S3400-N (Hitachi) scanning electron microscope, equipped with a Noran 7 dry EDX system (Thermo Scientific). An accelerating voltage of $15 \mathrm{keV}$ was used. For elemental mapping, the characteristic $\mathrm{x}$-ray lines of $\mathrm{Ca}(\mathrm{K}, 3.7$ $\mathrm{keV}), \mathrm{Eu}(\mathrm{L}, 5.9 \mathrm{keV}), \mathrm{Si}(\mathrm{K}, 1.7 \mathrm{keV})$ and $\mathrm{S}(\mathrm{K}, 2.3 \mathrm{keV})$ were taken. Cathodoluminescence light was collected and directed by means of an optic fiber to a ProEM1600 EMCCD camera attached to a Acton SP2300 monochromator (Princeton Instruments). EDX and CL spectra 
were gathered and stored simultaneously by mapping the imaged area in a 128-by-92 grid. Emission properties (such as peak maximum and emission intensity) were processed after the data collection. Temperature dependent CL measurements were obtained using a peltier heating and cooling stage (Deben), yielding effective sample temperatures ranging from 253 to $373 \mathrm{~K}$.

The temperature dependency of the photoluminescence was measured in a home-built setup in the range from $-50^{\circ} \mathrm{C}$ to $200^{\circ} \mathrm{C}$, using a cooling and heating stage. The excitation source was a near-UV LED (peaking at $400 \mathrm{~nm}$ ). The emitted light was directed to the Princeton detection setup, as described above.

\section{Results and discussion.}

Figure 1 shows the $x$-ray diffraction pattern for the synthesized phosphor. Based on the reference patterns of $\mathrm{Ca}_{2} \mathrm{SiS}_{4}$ and $\mathrm{CaEuSiS}_{4}$ (which has the same monoclinic crystallographic structure as $\mathrm{Eu}_{2} \mathrm{SiS}_{4}$ ), it is clear that both phases are present in the powder. From now on, the $\mathrm{Ca}_{2} \mathrm{SiS}_{4}$-like phase with relatively low europium concentration is called the C-phase, while the $\mathrm{Eu}_{2} \mathrm{SiS}_{4}$-like phase with about half of the europium ions replaced by calcium will be called the E-phase. Based on earlier work, it was estimated that the substitution of Ca by Eu in the C-phase is limited to 10 to $15 \%$, while the substitution of Eu by $\mathrm{Ca}$ in the E-phase is below 50 to $60 \%$ [13]. For the intended composition, a mixture of both phases can thus be expected.

Figure 2 shows the emission intensity of the phosphor as a function of temperature under excitation with a 400nm LED. Both the C- and E-phase show efficient absorption in this wavelength range [13]. The phosphor shows already a significant thermal quenching near room temperature, due to the elevated europium concentration. The thermal quenching can 
be described by the parameter $T_{0.5}$. This is the temperature at which the emission intensity is half of the maximum intensity (normally encountered at low temperature). For $\mathrm{Ca}_{2} \mathrm{SiS}_{4}: \mathrm{Eu}$ with low Eu concentration (1\% substitution) $\mathrm{T}_{0.5}$ is $470 \mathrm{~K}$ [20], which is considerably higher than for the present, heavily doped phosphor $\left(\mathrm{T}_{0.5}=350 \mathrm{~K}\right)$.

Besides the integrated emission intensity, also the emission spectrum was monitored during the thermal quenching measurement. Emission spectra, including the reflected fraction of the excitation by the 400nm-LED, are shown in the inset of Fig. 2 for selected temperatures. Based on all the acquired spectra, the full width at half maximum (FWHM) was calculated and displayed in Fig. 3. For increasing temperature, a broadening of the emission spectrum is expected, due to the occupation of higher vibrational levels. Although this general trend is indeed present, one also observes a deviation from the monotonic increase around $100^{\circ} \mathrm{C}$ (Fig. 3(a)). By evaluation of specific wavelengths (for which the emission intensity is $50 \%$ and $100 \%$ of the maximum emission intensity), we observe similar non-monotonic behaviour (Fig. 3(b)). To explain this, the emission properties of the phosphor at the microscopic level are discussed first.

Fig. 4 shows a backscattered electron image of a representative collection of agglomerated phosphor particles, along with chemical mappings for the key elements (Fig. 4(a)-(d)). The contrast in the backscattered image (Fig. $4(f)$ ) is mainly due to $Z$ contrast, and is reflected in the chemical EDX maps of Eu $(Z=63)$ and $\mathrm{Ca}(Z=20)$. From these two maps, a false colour image (Fig. 4(e)) is constructed, which clearly shows a phase separation. On the one hand, certain phosphor grains have the C-phase (with relatively low Eu concentration), while others show the E-phase (with moderate Ca concentration). This phase separation is also reflected in the cathodoluminescence spectra. Fig. $4(\mathrm{~g})$ shows the emission barycenter 
(calculated as $\left.\int I(\lambda) \lambda \mathrm{d} \lambda / \int I(\lambda) \mathrm{d} \lambda\right)$ for each point in the mapping. Based on these data, emission spectra from selected areas are shown in Fig. 4(h).

For the C-phase, the emission barycenter lies around $655 \mathrm{~nm}$. This phase shows two emission bands, peaking at 560 and $655 \mathrm{~nm}$, with the relative ratio depending on the europium concentration. For elevated Eu concentration (>2\%), energy transfer occurs and the emission spectrum is dominated by the red emission band, as can be seen in the emission spectrum for area 4 (Fig. $4(\mathrm{~g}, \mathrm{~h})$ ). For the E-phase, a single emission band peaking at $605 \mathrm{~nm}$ (area 2 ) is obtained. The position of this emission band is slightly dependent on the europium concentration, with a blue-shift for higher concentrations [13]. When comparing areas 1 and 2, one finds a relatively higher Eu concentration (Fig. 4(e)) in the former area, with a slight blue-shifted emission spectrum (Fig. 4(h)).

Although from the barycenter position it is clear that two separate phases are obtained, there are some small areas where the emission peaks at intermediate wavelengths (e.g. area 3). Careful analysis shows that this is due to small grains of both phases lying on top of or close to each other. For the used electron beam energy (15keV), the interaction volume is relatively large so that the electron beam simultaneously excites proximate grains.

To evaluate the thermal quenching behaviour at a local scale, the same type of mapping has to be performed at different temperatures. Then, maps showing the total emission intensity can be compared to each other (Fig. 5).

In order to obtain the thermal properties quantitatively, it is beneficial to divide mappings by each other. However, this requires a perfect alignment between mappings at different temperature, as otherwise too many artefacts are obtained, along with a reduced level of 
detail. Image distortions due to electrostatic charging or due to the use of the temperature stage were counteracted in the following way (Fig. 6). For two subsequent mappings, a topographical image was collected in line with the mapping of the $C L$, called $\mathrm{SE}_{\mathrm{A}}$ and $\mathrm{SE}_{\mathrm{B}}$. $\mathrm{By}$ comparing both images, it is clear that some shifting and distortions occurred, which is shown schematically in Fig. 6. By defining displacement vectors for the edges of the image (illustrated by the red arrows), the image $\mathrm{SE}_{\mathrm{B}}$ is transformed to image $\mathrm{SE}_{\mathrm{B}}{ }^{\prime}$ to show as much overlap as possible with the original image $\mathrm{SE}_{\mathrm{A}}$. These displacement vectors can then be used to transform the $\mathrm{CL}$ maps in the same way. Obviously, a mask has to be defined for areas which do not contain data (dark blue area in Fig. 6).

Fig. 5 (a) and (b) show two mappings of the total $\mathrm{CL}$ intensity at $-20^{\circ} \mathrm{C}$ and $100^{\circ} \mathrm{C}$, respectively. Fig. 5(b) was already transformed in such a way that the backscattered electron images overlap. At the higher temperature, the total emission intensity has already significant decreased, in line with the macroscopic (PL) behaviour (Fig. 2). Note that the same intensity scale was used for Figs. 5 (a) and (b). Now both images can be divided pointwise to assess the local thermal quenching behaviour (Fig. 5(c)). Although some artefacts are present due to the data processing, this map shows a reliable and detailed picture of the thermal quenching behaviour at the microscopic level. Large differences can be seen in this ratio for the different grains, pointing at a locally different thermal quenching behaviour. This can now be compared to the occurrence of the two phases in Fig. 5(d). In this map, the fraction of the emission intensity in the range from $550 \mathrm{~nm}$ to $600 \mathrm{~nm}$ is displayed. Low values ( 0 to 0.1 ) point at the $\mathrm{C}$-phase, while higher values $(>0.25)$ point at the E-phase. For high Eu concentrations within the E-phase, the value reaches 0.4 , due to the blue-shift of the spectrum. We can now conclude that the thermal quenching is considerably worse for the Ephase than for the C-phase. If the E-phase contains a high europium concentration (e.g. area 
1 in Fig. $4(\mathrm{~g}))$, then the emission has almost vanished at $100^{\circ} \mathrm{C}$. The changes in the emission spectrum as a function of temperature (Fig.3) can now be interpreted. Increasing temperatures lead to a reduction in the emission intensity for both phases. However, the thermal quenching is much stronger for the E-phase (with some variability depending on the local Eu concentration), so that it almost entirely quenches out around $100^{\circ} \mathrm{C}$. Therefore, the contribution of the emission of the E-phase (peaking around 600nm) reduces more drastically compared to the emission of the C-phase (peaking around 650nm). Consequently, the global emission spectrum shows a red shift at around $100^{\circ} \mathrm{C}$, especially for the high energy side of the emission band. In addition, the emission spectrum (for both phases) is broadening at higher temperatures. Finally we can compare the emission spectra for both phases as a function of temperature at a point-wise level or for selected phosphor particles. This is illustrated in Fig. 7, which shows the CL emission spectra of the selected areas in Fig. $4(\mathrm{~g})$ as a function of temperature.

The spectra for each area were normalized to the emission intensity at $253 \mathrm{~K}$, so that the thermal behaviour can be evaluated for each area, in terms of emission intensity (i.e. the thermal quenching) and spectral changes. Typical parameters (the integrated emission intensity, the emission peak wavelength and the FWHM) are derived from the spectra and shown in Table 1.

\begin{tabular}{c|cccc|ccc} 
& \multicolumn{3}{|c|}{} & \multicolumn{3}{c}{$\lambda_{\text {peak }}$ (FWHM) in nm } \\
T(K) & Area 1 & Area 2 & Area 4 & PL-bulk & Area 1 & Area 2 & Area 4 \\
\hline 253 & 1000 & 1000 & 1000 & 1000 & $604(69)$ & $611(69)$ & $656(87)$ \\
323 & 500 & 724 & 825 & 756 & $598(79)$ & $607(77)$ & $651(96)$ \\
373 & 163 & 275 & 503 & 433 & $596(94)$ & $603(86)$ & $645(105)$
\end{tabular}


Table 1. Spectral data derived from the CL spectra as shown in Fig. 7. "PL-bulk" refers to the photoluminescence emission intensity as shown in Fig. 2.

These data confirm the earlier statements. The C-phase (area 4) has the highest thermal quenching temperature. For the E-phase (area 1 and 2), the thermal quenching is worse, with the exact behaviour depending on the europium concentration. For each phase, the emission peak shifts slightly towards shorter wavelength at higher temperature, and the FWHM increases. One also observes that the $(C L)$ thermal quenching behaviour for the separate areas is in line with the (PL) thermal quenching of the bulk phosphor, when considering that the bulk phosphor consists of a mixture of both $\mathrm{C}$ and E-phases. Finally, the temperature dependent changes in the emission spectrum as observed for the bulk phosphor can now be explained (Fig. 3). When the temperature increases from $-20^{\circ} \mathrm{C}$ to $50^{\circ} \mathrm{C}$, the position of the emission peak is rather steady. This is a result of the blue shift of the emission maximum in both phases for increasing temperature, being counteracted by the slightly strongly thermal quenching of the E-phase, reducing the contribution on the shorter wavelength side. In the range from $50^{\circ} \mathrm{C}$ to $100^{\circ} \mathrm{C}$ the intensity of the E-phase drops much faster than this for the C-phase, which leads to a red-shift of the emission peak. Above $100^{\circ} \mathrm{C}$, the emission is mainly determined by the C-phase, leading to a blue-shift upon increasing temperature. A similar reasoning can be used to explain the temperature dependency of the FWHM for the bulk phosphor.

\section{Conclusions.}

In this work the thermal quenching behaviour of a multi-phase $(\mathrm{Ca}, \mathrm{Eu})_{2} \mathrm{SiS}_{4}$ phosphor was studied, both on a macroscopic and a microscopic level. Two phases are present in the material. First, a $\mathrm{Ca}_{2} \mathrm{SiS}_{4}$-like phase (with the main emission band around $655 \mathrm{~nm}$ ), has a 
relatively high thermal quenching temperature. Second, $\mathrm{Eu}_{2} \mathrm{SiS}_{4}$-like phases are present with some variations in composition, with an emission spectrum peaking around $605 \mathrm{~nm}$. This phase shows a much lower thermal quenching temperature. The different temperature dependency is reflected in the emission properties when measuring the (photo)luminescence for the bulk phosphor.

The presently studied dopant concentration in this thiosilicate phosphor is obviously not the most optimum for application as conversion phosphor in LEDs, due to the relatively low associated thermal quenching temperature and the broadness of the emission spectrum. However, the main purpose of this paper was to show the additional information which can be obtained by an evaluation of the thermal quenching at the microscopic level. In that sense, cathodoluminescence spectroscopy gives an added value in understanding the underlying emission behaviour of multi-phase phosphors. After correcting for image distortions, it is possible to quantitatively compare cathodoluminescence intensities and emission spectra as a function of temperature. This technique can easily be applied to other phosphor systems as well, e.g. to detect impurity phases, to assess dopant segregation and to study the influence of grain boundaries.

\section{Acknowledgments.}

The authors would like to thank Lorenzo Degryse for assistance in preparing the samples. JB is grateful to the BOF-UGent for financial support. 


\section{References.}

[1] P. F. Smet, A. B. Parmentier, D. Poelman, Journal of the Electrochemical Society 158 (2011) R37-R54.

[2] R. J. Xie, N. Hirosaki, Y. Q. Li, T. Takeda, Materials 3 (2010) 3777-3793.

[3] P. Dorenbos, Journal of Luminescence 104 (2003) 239-260.

[4] R. J. Xie, N. Hirosaki, Science and Technology of Advanced Materials 8 (2007) 588-600.

[5] K. Uheda, N. Hirosaki, Y. Yamamoto, A. Naito, T. Nakajima, H. Yamamoto, Electrochemical and Solid State Letters 9 (2006) H22-H25.

[6] Y. Q. Li, J. E. J. van Steen, J. W. H. van Krevel, G. Botty, A. C. A. Delsing, F. J. DiSalvo, G. de With, H. T. Hintzen, Journal of Alloys and Compounds 417 (2006) 273-279.

[7] R. J. Xie, N. Hirosaki, N. Kimura, K. Sakuma, M. Mitomo, Applied Physics Letters 90 (2007) 191101.

[8] P. F. Smet, I. Moreels, Z. Hens, D. Poelman, Materials 3 (2010) 2834-2883.

[9] A. B. Parmentier, P. F. Smet, D. Poelman, Optical Materials 33 (2010) 141-144.

[10] P. F. Smet, K. Korthout, J. E. Van Haecke, D. Poelman, Materials Science and Engineering BSolid State Materials for Advanced Technology 146 (2008) 264-268.

[11] Y. Nanai, C. Sasaki, Y. Sakamoto, T. Okuno, Journal of Physics D-Applied Physics 44 (2011) 405402.

[12] A. B. Parmentier, P. F. Smet, F. Bertram, J. Christen, D. Poelman, Journal of Physics D-Applied Physics 43 (2010) 085401.

[13] P. F. Smet, N. Avci, B. Loos, J. E. Van Haecke, D. Poelman, Journal of Physics-Condensed Matter 19 (2007) 246223.

[14] J. Olivierfourcade, M. Ribes, E. Philippot, P. Merle, M. Maurin, Materials Research Bulletin 10 (1975) 975-982.

[15] M. Nishimura, Y. Nanai, T. Bohda, T. Okuno, Japanese Journal of Applied Physics 48 (2009) 072301. 
[16] M. Sugiyama, Y. Nanai, Y. Okada, T. Okuno, Journal of Physics D-Applied Physics 44 (2011) 095404.

[17] B. Bastek, O. August, T. Hempel, J. Christen, M. Wieneke, J. Blasing, A. Dadgar, A. Krost, U. Wendt, Applied Physics Letters 96 (2010) 172102.

[18] M. F. Hamers, M. R. Drury, Meteoritics \& Planetary Science 46 (2011) 1814-1831.

[19] H. Niioka, T. Furukawa, M. Ichimiya, M. Ashida, T. Araki, M. Hashimoto, Applied Physics Express 4 (2011) 112402.

[20] P. F. Smet, N. Avci, D. Poelman, Journal of the Electrochemical Society 156 (2009) H243$\mathrm{H} 248$. 
Fig. 1. x-ray diffraction pattern with reference diffraction positions for (a) $\mathrm{CaEuSiS}_{4}$ and (b) $\mathrm{Ca}_{2} \mathrm{SiS}_{4}$ indicated at the top of the figure.

Fig. 2: PL intensity as function of temperature. The inset shows the emission spectra (including the reflected light from the LED at $400 \mathrm{~nm}$ ) at selected temperatures $\left(-50^{\circ} \mathrm{C}, 0^{\circ} \mathrm{C}\right.$, $50^{\circ} \mathrm{C}, 100^{\circ} \mathrm{C}, 150^{\circ} \mathrm{C}$ ), as indicated in the main figure with the coloured dots.

Fig.3: (a) Influence of temperature on the FWHM of the emission spectrum. (b) Influence of temperature on the peak wavelength $\left(\lambda_{m}\right)$ and the wavelengths at half maximum $\left(\lambda_{L}\right.$ and $\left.\lambda_{R}\right)$, as indicated in the inset.

Fig.4: (a-d) Chemical maps obtained by EDX for (a) Si-K, (b) S-K, (c) Ca-K, (d) Eu-L. (e) false colour image, with red and green coding for Ca-K and Eu-L, respectively. (f) Backscattered electron image, (g) map of the CL emission barycenter (see text), (h) CL emission spectra at $20^{\circ} \mathrm{C}$ for the areas indicated in (g). The imaged area measures 27.4 by $19.7 \mu \mathrm{m}$.

Fig. 5: Total emission intensity for the sample shown in Fig. $4(\mathrm{f})$ at (a) $-20^{\circ} \mathrm{C}$ and (b) $100^{\circ} \mathrm{C}$. (c) Emission intensity at $100^{\circ} \mathrm{C}$ divided by this at $-20^{\circ} \mathrm{C}$. (d) Fraction of the emission spectrum in the range from 550 to $600 \mathrm{~nm}\left(\right.$ at $-20^{\circ} \mathrm{C}$ ).

Fig. 6. Schematic description of the resampling method to correct for image shifts during the recording of $C L$ maps at two temperatures $A$ and $B$, based on the topographical images $S_{A}$ and $\mathrm{SE}_{\mathrm{B}}$. Displacement vectors (red) are used to transform $\mathrm{SE}_{\mathrm{B}}$ to $\mathrm{SE}_{\mathrm{B}}{ }^{\prime}$, to maximize the overlap between $\mathrm{SE}_{\mathrm{A}}$ and $\mathrm{SE}_{\mathrm{B}}{ }^{\prime}$.

Fig. 7. CL emission spectra as a function of temperature for three selected areas, as indicated in the inset (see also Fig. 4(g)). Spectra were normalized for all areas to the emission intensity at $253 \mathrm{~K}$. 


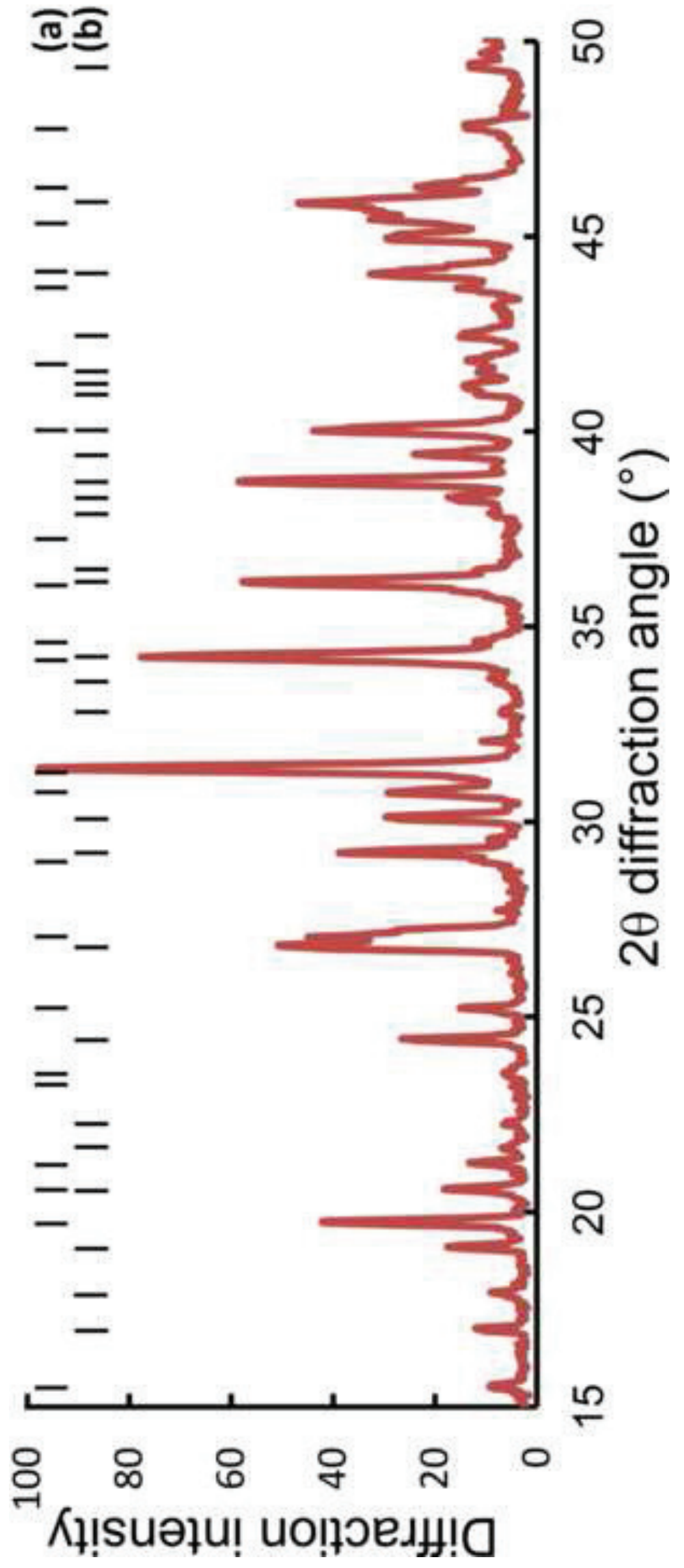

ఏ

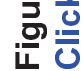




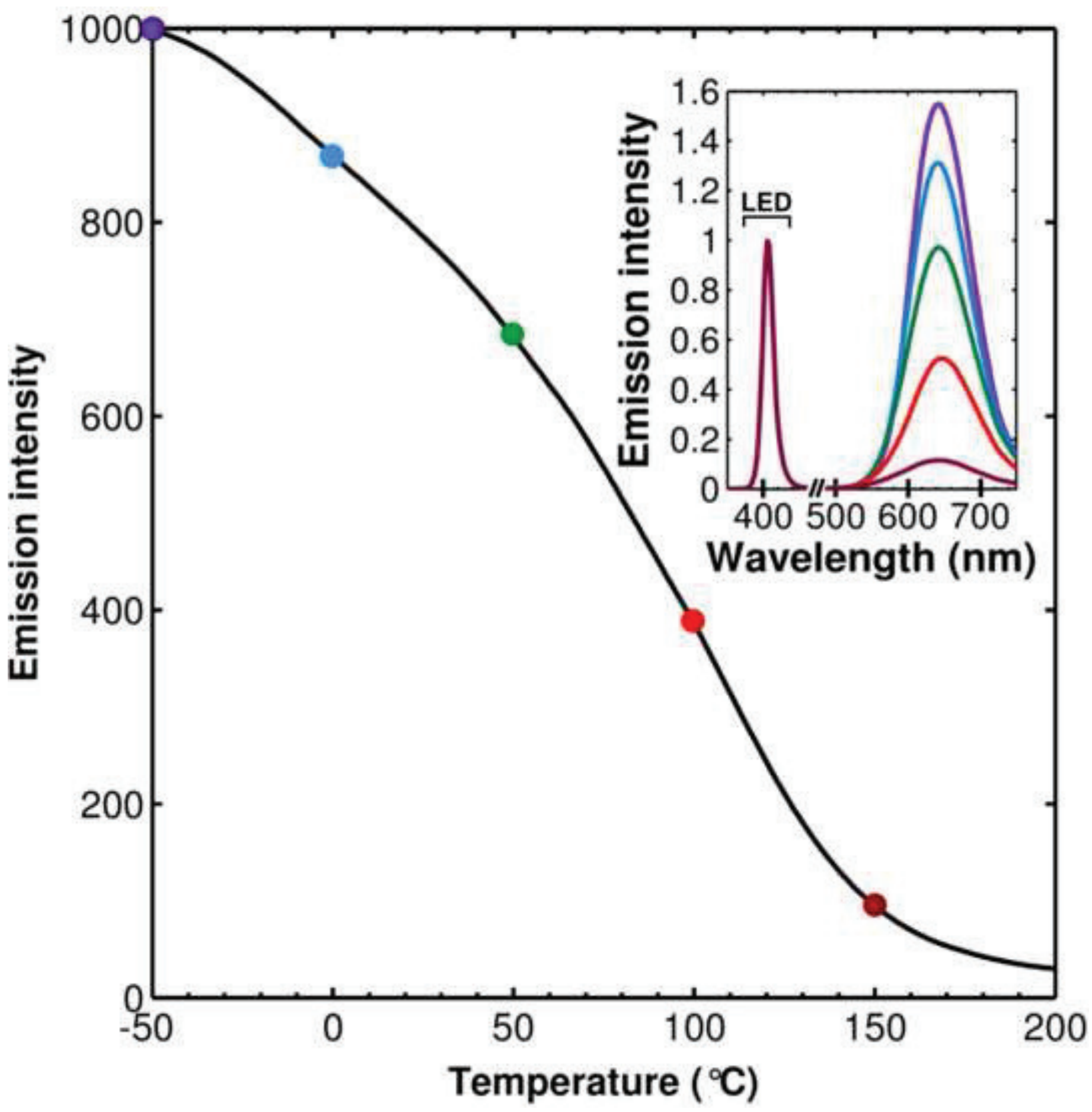



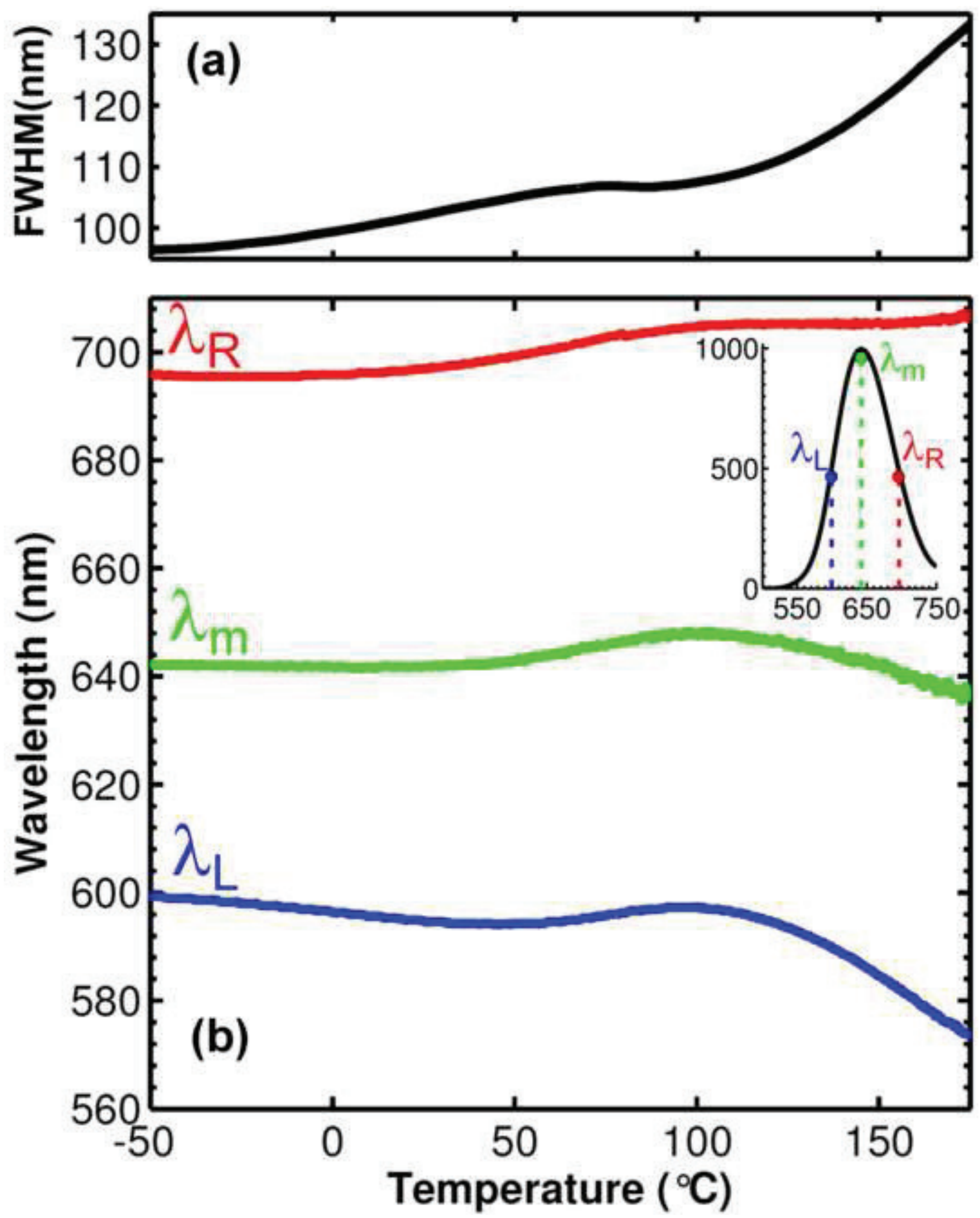

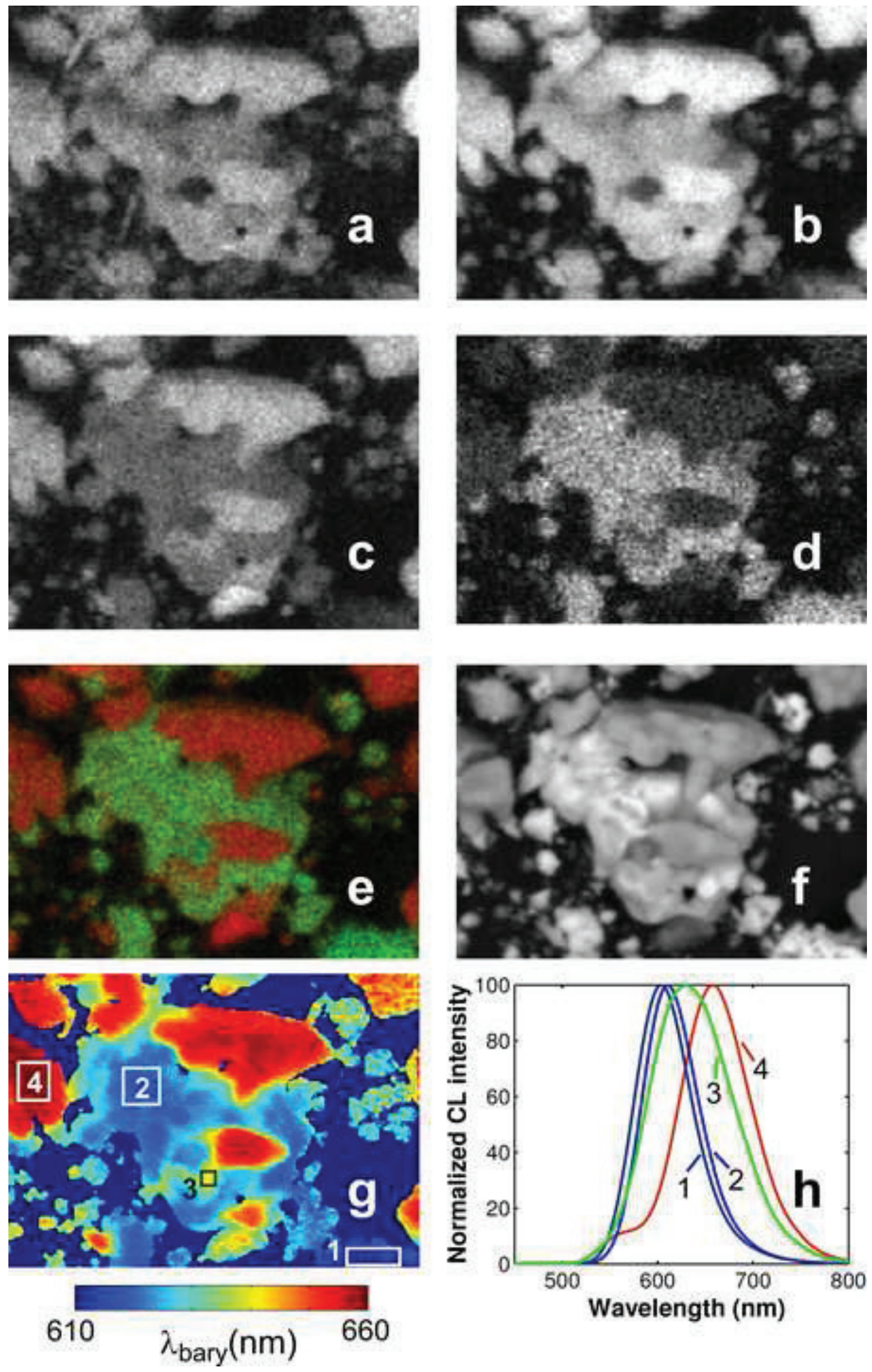

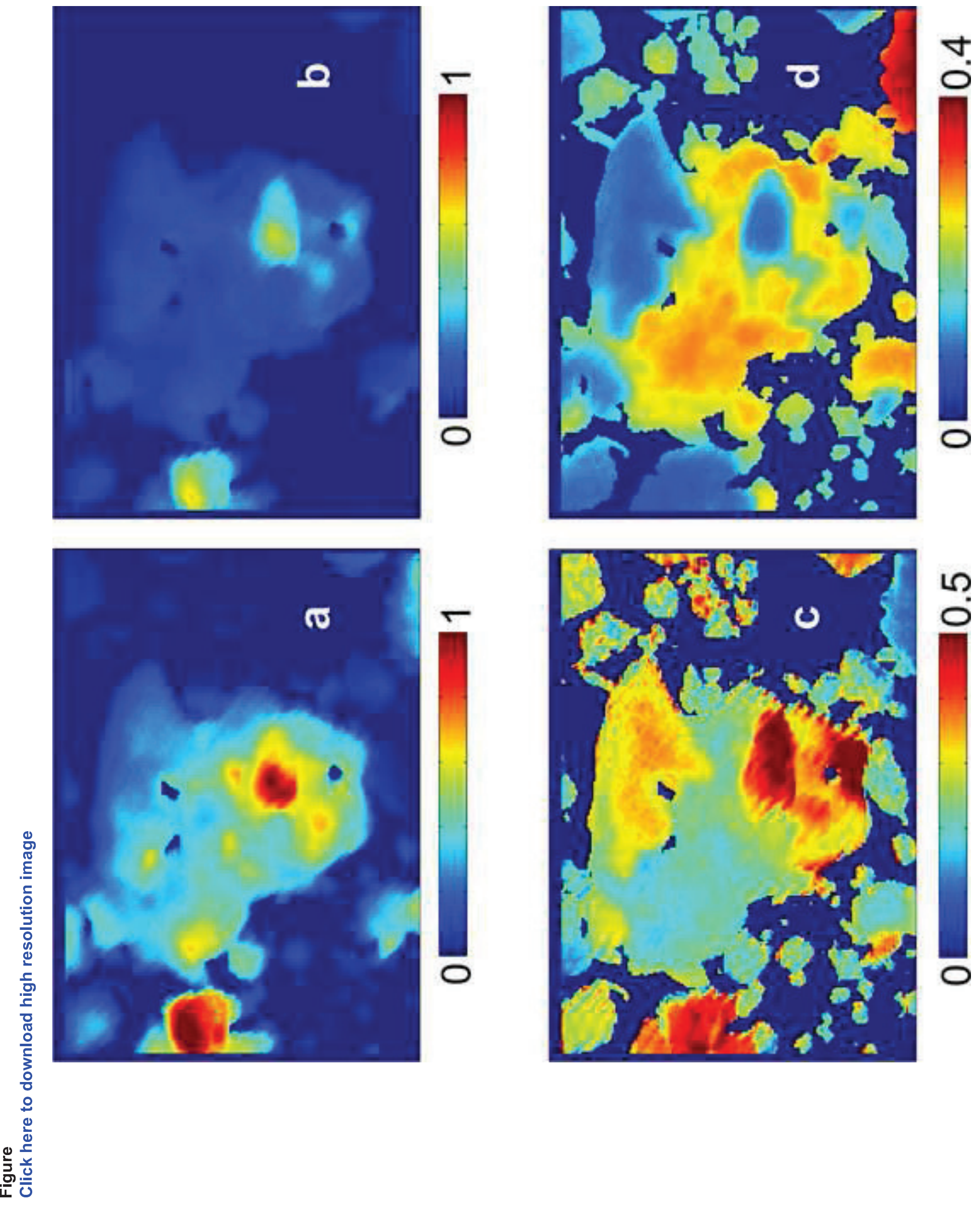

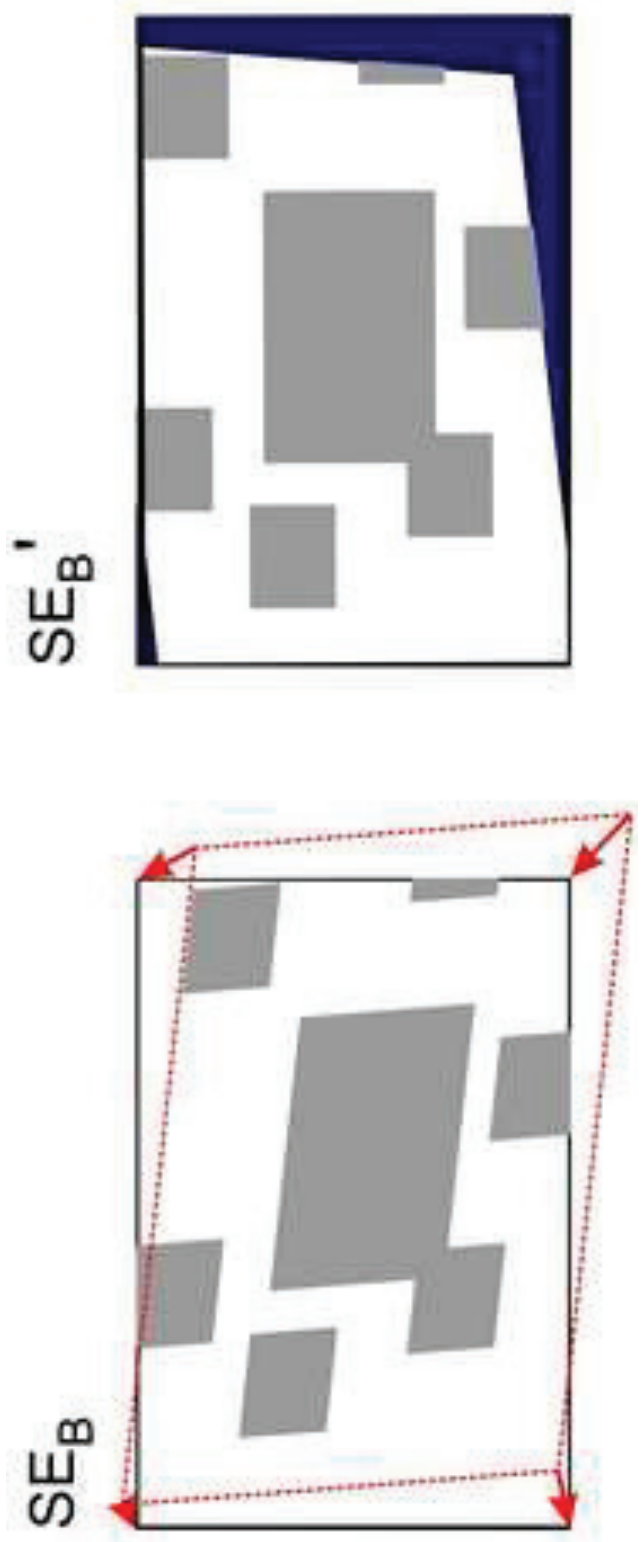

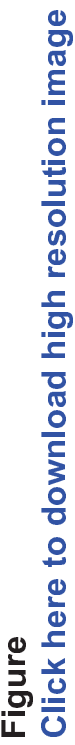

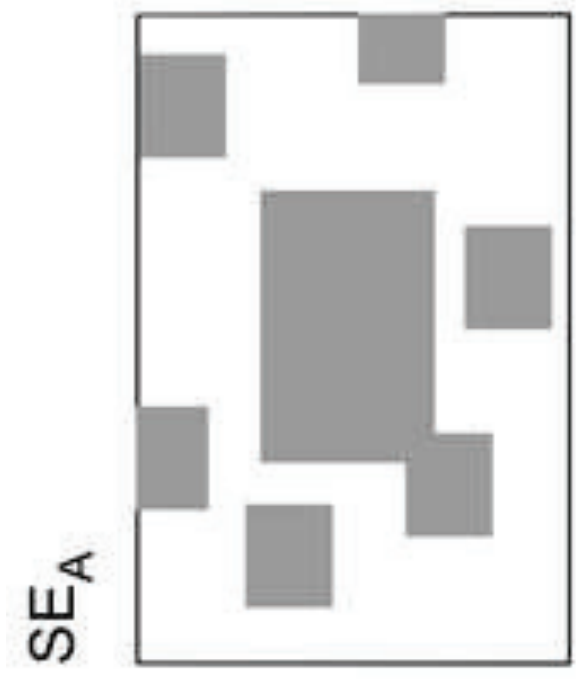



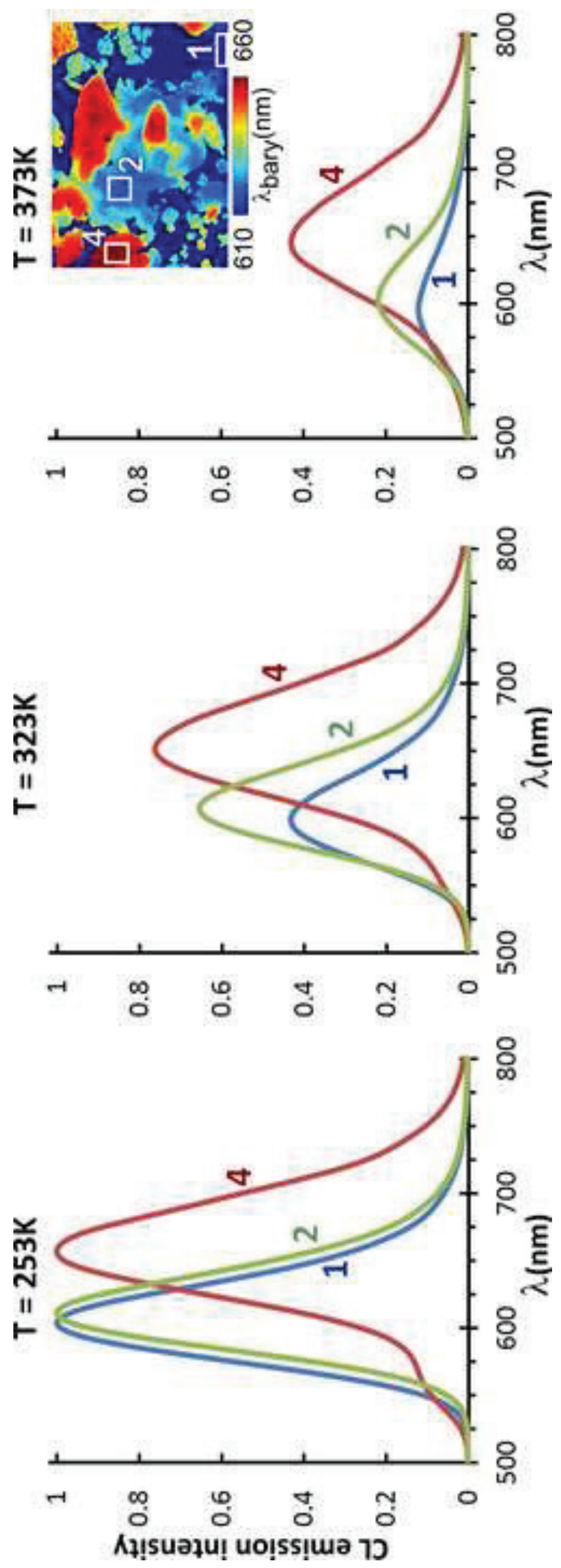

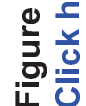

